Acta Crystallographica Section F

Structural Biology

and Crystallization

Communications

ISSN 1744-3091

\section{Jun $\mathrm{Yi}^{*}$, Leonard M. Thomas and George B. Richter-Addo}

Department of Chemistry and Biochemistry, University of Oklahoma, 101 Stephenson

Parkway, Norman, OK 73019, USA

Correspondence e-mail: yijun@ou.edu

Received 5 December 2010

Accepted 4 April 2011

PDB Reference: R-state aquomethemoglobin, $3 p 5 q$.

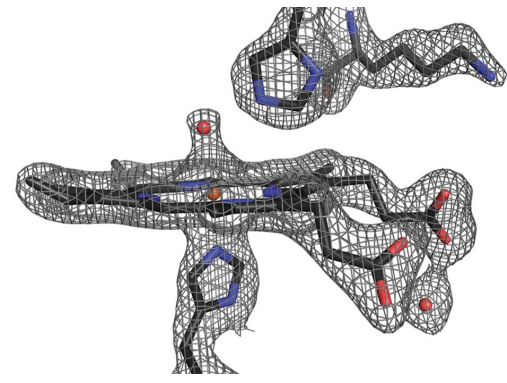

(C) 2011 International Union of Crystallography All rights reserved

\section{Structure of human R-state aquomethemoglobin at $2.0 \AA$ resolution}

The crystal structure of tetrameric $(\alpha \beta)_{2}$ R-state human adult aquomethemoglobin is reported at $2.0 \AA$ resolution. The asymmetric unit contained one $\alpha \beta$ subunit pair. The R-state crystal belonged to space group $P 4_{1} 2{ }_{1} 2$, with unit-cell parameters $a=b=53.6, c=192.8 \AA$. An Fe-bound water molecule was modeled into the heme distal pockets of each of the $\alpha$ and $\beta$ subunits. In the $\alpha$ subunit, a highly ordered liganded water was modeled with an $\mathrm{Fe}-\mathrm{O}$ (water) distance of $2.2 \AA$ and appears to be protected against escape from the distal pocket by the conformation of the heme propionate groups, which point upwards towards the distal His58 residue aided by a hydrogen-bonding network involving the solvent. In the $\beta$ subunit, the liganded water exhibited greater motion and was modeled with a longer $\mathrm{Fe}-\mathrm{O}$ (water) distance of $2.5 \AA$; in this subunit both propionate groups point downwards away from the distal His63 residue, presumably allowing greater motion of the liganded water in and out of the distal pocket.

\section{Introduction}

Hemoglobin $(\mathrm{Hb})$ is an essential blood protein that in the ferrous form binds dioxygen in the lungs and transports it to the muscle protein myoglobin $(\mathrm{Mb})$. Ferrous $\mathrm{Hb}$ (i.e. $\mathrm{HbFe}^{\mathrm{II}}$ ) is the active form for the binding of dioxygen and can be converted to the ferric form $\left(\mathrm{metHb} ; \mathrm{HbFe}^{\mathrm{III}}\right)$ by oxidation either through autoxidation or by the use of oxidants such as nitrite (Titov \& Petrenko, 2005) and arylhydroxylamines (Kiese \& Plattig, 1959). Importantly, metHb is unable to bind dioxygen. Ferrous $\mathrm{Hb}$ is the prevalent form of $\mathrm{Hb}$ under normal physiological conditions and is kept in the reduced form by the hemoglobin reductase enzyme system (Rockwood et al., 2003; Mauk \& Mauk, 1982). However, it has been determined that up to $3 \%$ metHb can also be present under normal physiological conditions.

Several quaternary states of $\mathrm{Hb}$ have been defined in the literature and an excellent review of $\mathrm{Hb}$ conformational states has been provided by Yonetani \& Laberge (2008). The most widely recognized states are the tense ( $\mathrm{T}$; least reactive, low affinity) and relaxed (R; most reactive, high affinity) conformational states as first proposed by Monod, Wyman and Changeux in 1965 as part of their two-state concerted model for allostery (Monod et al., 1965); these are also commonly associated with deoxy [i.e. five-coordinate heme $\mathrm{Fe}$ with no (sixth) axial ligand] and oxy (i.e. six-coordinate heme Fe) heme centers, respectively. However, six-coordinate T-state structures such as T-state $\mathrm{HbNO}$, obtained by soaking the NO ligand into crystals of T-state $\mathrm{Hb}$, have been reported (Chan et al., 2004). Additional quaternary states of $\mathrm{Hb}$ such as the RR2, R3 and R2 states have been reported in recent years as either intermediates or end-state structures (Safo \& Abraham, 2003, 2005).

Fifty years ago, horse oxyHb was the first three-dimensional crystal structure of $\mathrm{Hb}$ to be solved (Perutz et al., 1960) and a $2.0 \AA$ resolution structure has been published (Ladner et al., 1977). Arnone and coworkers reported the $1.6 \AA$ resolution crystal structure of horse Rstate metHb (Robinson et al., 2003). In this latter structure, liganded water molecules were bound to Fe in both the $\alpha$ and $\beta$ subunits of the tetrameric protein. The crystal structure of T-state human 
Table 1

X-ray data-collection and refinement statistics.

Values in parentheses are for the highest resolution shell.

\begin{tabular}{ll}
\hline Data collection & \\
Space group & $P 4_{1} 2_{1} 2$ \\
Unit-cell parameters $(\AA)$ & $a=b=53.5, c=192.8$ \\
Wavelength $(\AA)$ & 1.5418 \\
Temperature $(\mathrm{K})$ & 100 \\
Resolution range $(\AA)$ & $18.14-2.00(2.05-2.00)$ \\
No. of observations & 114154 \\
Unique reflections & 19844 \\
Average multiplicity & $5.75(6.02)$ \\
Completeness $(\%)$ & $99.8(100)$ \\
$\langle I / \sigma(I)\rangle$ & $9.3(3.2)$ \\
$R_{\text {merge }}^{\dagger}$ & $0.075(0.433)$ \\
Refinement & \\
No. of protein atoms & 2254 \\
No. of heteroatoms & 128 \\
$R$ factor $\ddagger$ & 0.221 \\
$R_{\text {free }}$ & 0.267 \\
Average $B$ factor $\uparrow\left(\AA^{2}\right)$ & 39.0 \\
R.m.s. deviations $\dagger \dagger$ & \\
Bond lengths $(\AA)$ & 0.01 \\
Bond angles $\left({ }^{\circ}\right)$ & 1.03 \\
Ramachandran plot & \\
Favored & \\
Outliers & 98.2 \\
Rotamer outliers & 0 \\
PDB code & 0 \\
\hline
\end{tabular}

$\dagger R_{\text {merge }}=\sum_{h k l} \sum_{i}\left|I_{i}(h k l)-\langle I(h k l)\rangle\right| / \sum_{h k l} \sum_{i} I_{i}(h k l)$, where $I_{i}(h k l)$ is the $i$ th used observation for unique $h k l$ and $\langle I(h k l)\rangle$ is the mean intensity for unique $h k l$. $\neq R=$ $\sum_{h k l}|| F_{\text {obs }}|-| F_{\text {calc }}|| / \sum_{h k l}\left|F_{\text {obs }}\right|$, where $F_{\text {obs }}$ and $F_{\text {calc }}$ are the observed and calculated structure factors, respectively. $\& R_{\text {free }}$ was calculated using $5 \%$ of the randomly selected diffraction data which were excluded from refinement. The average for the polypeptide atoms. †† Deviations from ideal values (Engh \& Huber, 1991). 枺 Calculated using MolProbity as implemented in PHENIX (Chen et al., 2010).

aquometHb at $2.1 \AA$ resolution has been reported (Liddington et al., 1992). Surprisingly, however, no crystal structure of R-state human aquometHb has been reported. A $3.2 \AA$ resolution structure of human metHb has been deposited in the Protein Data Bank (PDB entry 1jy7; Biswal \& Vijayan, 2002); at this resolution, water was not added to the final model. Furthermore, the asymmetric unit was determined to contain three independent quaternary states that lie between the $\mathrm{R}$ and $\mathrm{R} 2$ conformational states. In this article, we report the crystal structure of R-state human aquometHb at $2.0 \AA$ resolution.

\section{Materials and methods}

\subsection{Purification and crystallization}

The metHb protein $\left(\lambda_{\max }=405 \mathrm{~nm}\right)$ was prepared by oxidation of human oxyHb $\left(\lambda_{\max }=415 \mathrm{~nm}\right)$ using excess potassium ferricyanide in $10 \mathrm{~m} M$ sodium phosphate $\mathrm{pH} 7.0$ followed by dialyzing this mixture against sodium phosphate $\mathrm{pH} 6.8$ to remove excess ferricyanide and other small inorganics. Crystals of human metHb were obtained using the batch method in a manner similar to that employed to obtain crystals of the ferric Hb-nitrite complex (Yi et al., 2008). $100 \mu \mathrm{l}$ protein solution (at $4-30 \mathrm{mg} \mathrm{ml}^{-1}$ ) was added to $0.32 \mathrm{ml} \mathrm{3.2-3.4} \mathrm{M}$ phosphate buffer $\mathrm{pH} 6.8$ and a few drops of toluene were then added. Crystals of metHb grew to a reasonable size after a few days and were harvested in cryoloops and soaked in mother liquor containing $16 \%$ glycerol for $2 \mathrm{~min}$. The harvested crystals were flash-frozen in liquid nitrogen and stored for data collection.

\subsection{Data collection and processing}

$\mathrm{X}$-ray diffraction data were collected from aquometHb crystals at $100 \mathrm{~K}$ on a Rigaku RU-H3R X-ray generator operated at $50 \mathrm{kV}$ and
$100 \mathrm{~mA}$ using $\mathrm{Cu} K \alpha$ radiation $(\lambda=1.5418 \AA)$. Diffraction data were detected using an R-AXIS IV $^{++}$image-plate detector system with the crystal-to-detector distance set to $135 \mathrm{~mm}$ and an exposure time of 6 min per image. 80 diffraction images were collected using $1^{\circ}$ oscillation increments. Diffraction data were processed and scaled with the $d^{*} T R E K$ program (Macintosh v.99D; Pflugrath, 1999).

\subsection{Structure solution and refinement}

The molecular-replacement method (MOLREP as implemented in the CCP4 package; Vagin \& Teplyakov, 2010; Winn et al., 2011) was used to solve the phase problem using the $1.25 \AA$ resolution structure of human HbCO (PDB code 1ird; S.-Y. Park \& J. R. H. Tame, unpublished work) as a search model with the $\mathrm{CO}$ and solvent molecules removed from the model. Structural refinement was carried out using the CCP4 program REFMAC (Murshudov et al., 2011) and the program PHENIX (Adams et al., 2002). For the $R_{\text {free }}$ calculation, a randomly selected $5 \%$ of the reflections were flagged

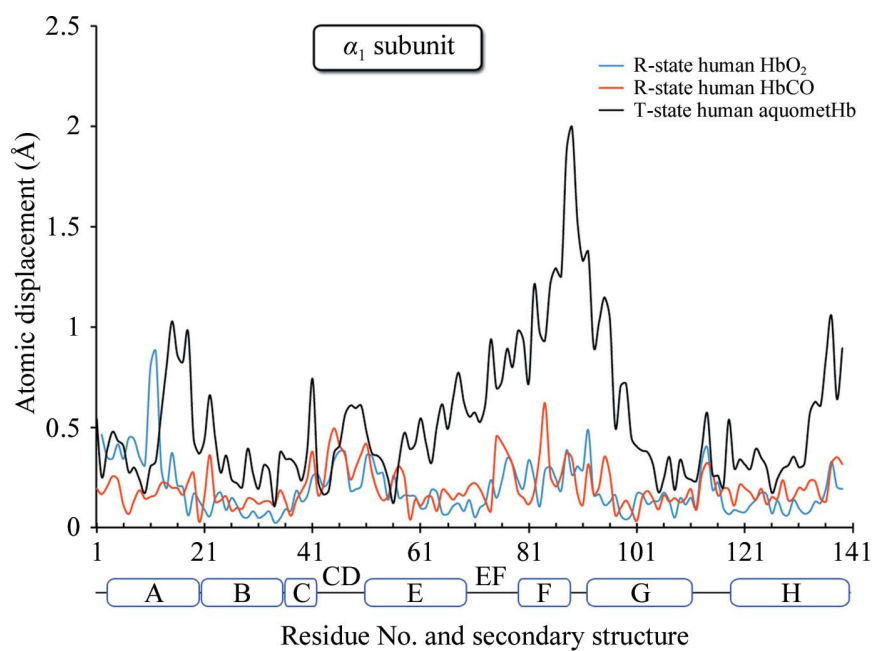

(a)

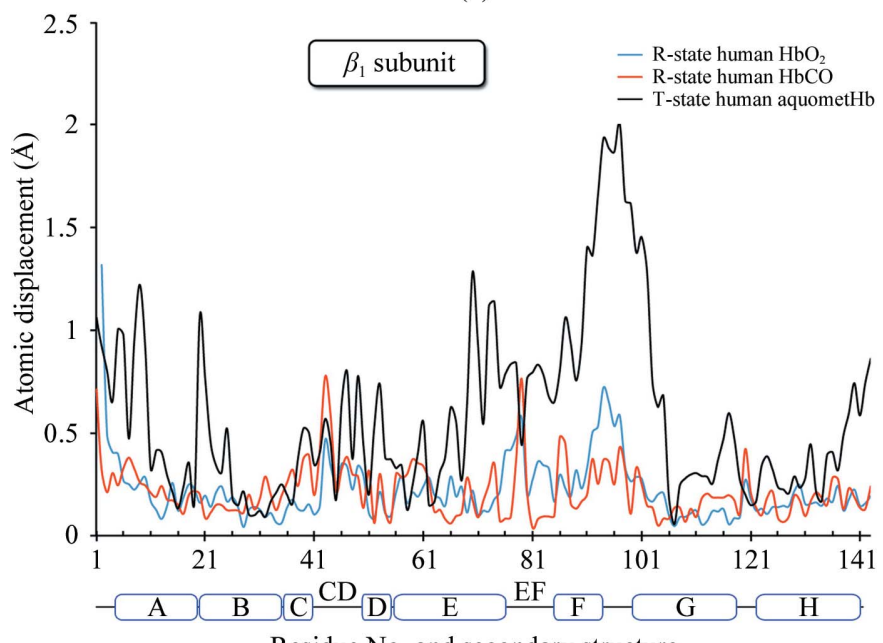

Residue No. and secondary structure

(b)

Figure 1

Plots of the $\mathrm{C}^{\alpha}$ displacements (in $\AA$ ) as a function of residue number in the $\alpha_{1}(a)$ and $\beta_{1}(b)$ subunits. The R-state aquometHb structure (this work) is used as the reference structure. Atomic displacements are shown for the R-state $\mathrm{HbO}_{2}$ structure (blue trace, PDB entry $2 \mathrm{dn} 1,1.25 \AA$ resolution, space group $P 4_{1} 2_{1} 2$; Park et al., 2006), the R-state $\mathrm{HbCO}$ structure (red trace, PDB entry 1ljw, $2.16 \AA$ resolution, space group $P 4_{1} 2_{1} 2$; Safo et al., 2002) and the T-state human aquometHb structure (black trace, PDB entry $1 \mathrm{hgb}, 2.1 \AA$ resolution, space group $P 2_{1} 2_{1} 2_{1}$; Liddington et al., 1992). 
and carried throughout the complete refinement procedure. Bulksolvent modeling and isotropic scaling of the observed and calculated structural amplitudes were used during the restrained refinements. Three toluene molecules were added into the crystal lattice based on density observed in an $F_{\mathrm{o}}-F_{\mathrm{c}}$ difference electron-density map. The C-terminal residues Lys140 and Arg141 in the $\alpha$ subunit were omitted from the structural model owing to a lack of defined electron density. Water molecules were added using the 'Update Waters' command in $P H E N I X$. The final crystallographic $R$ and $R_{\text {free }}$ values for the model are 0.221 and 0.267 , respectively, in the $18.1-2.0 \AA$ resolution range. The weighted map coefficient from REFMAC was imported into $F F T$ to generate the $2 F_{\mathrm{o}}-F_{\mathrm{c}}$ electron-density maps and $F_{\mathrm{o}}-F_{\mathrm{c}}$ difference electron-density maps. Data-processing and structurerefinement statistics are shown in Table 1 . Figures were drawn using PyMOL (DeLano, 2002) and labels were added using Adobe Photoshop.

\section{Results and discussion}

3.1. Overall structure of the R-state human tetrameric aquometHb protein

Over 200 human $\mathrm{Hb}$ crystal structures have been solved and deposited in the PDB (as of September 2010). Surprisingly, however, no R-state human aquometHb structure has been reported.

The human aquometHb crystal structure reported here contains one $\alpha \beta$ subunit pair in the asymmetric unit. The diffraction data and refinement statistics are summarized in Table 1 . The crystal belonged to space group $P 4_{1} 22$, with unit-cell parameters $a=b=53.6$, $c=192.8 \AA$.

It is important to note that the $P 4_{1} 2_{1} 2$ space group is frequently encountered in R-state Hb structures (Safo \& Abraham, 2003). We superimposed the $\mathrm{C}^{\alpha}$ atoms of the aquometHb structure reported here with those of the previously reported R-state human $\mathrm{Hb}$ derivatives $\mathrm{HbCO}$ (Safo et al., 2002) and $\mathrm{HbO}_{2}$ (Park et al., 2006) using the $\alpha_{1} \beta_{1}$ pair for the alignment. The r.m.s.d.s calculated using the $L S Q K A B$ program in CCP4 between the $\mathrm{C}^{\alpha}$ chains of the $\alpha_{1} \beta_{1}$ subunits in the quaternary structures of R-state human aquometHb (this work) and those of R-state human $\mathrm{HbCO}$ and $\mathrm{HbO}_{2}$ are 0.30 and $0.33 \AA$, respectively. The results are shown in Fig. 1 .

There is good overlap of the $\mathrm{C}^{\alpha}$ atoms among these three tetrameric structures. However, the overlap of the $\mathrm{C}^{\alpha}$ chains of the $\alpha_{1} \beta_{1}$ pairs of our human aquometHb structure (this work) and the reported T-state human aquometHb (Liddington et al., 1992) is not as good; the r.m.s.d. for the $\mathrm{C}^{\alpha}$ chains for the $\alpha_{1} \beta_{1}$ subunits is $0.68 \AA$, which is larger than the r.m.s.d.s for the other R-state $\alpha_{1} \beta_{1}$ overlays shown in Fig. 1. These results are consistent with our description of our human aquometHb structure (this work) as being in the R-state conformation.

3.2. The heme distal pockets: axial water coordination and heme propionate orientations

The $2 F_{\mathrm{o}}-F_{\mathrm{c}}$ electron-density maps and final models of the individual $\alpha$ and $\beta$ heme sites are shown in Fig. 2.

There are several interesting features present in this human $\mathrm{R}$-state aquometHb crystal structure. Firstly, the propionate groups of the prosthetic heme moiety in the $\alpha$ subunit show a 'locked' conformation (propionates pointing 'upwards' towards the distal His58 residue); in contrast, the propionate groups in the $\beta$ subunit show an 'open' conformation (propionates pointing downwards away from the analogous distal His63 residue). Secondly, the $\mathrm{Fe}-\mathrm{O}$ (water) distance of $2.5 \AA$ in the $\beta$ subunit is longer than that in the $\alpha$ subunit at $2.2 \AA$; interestingly, however, the calculated distances between the $\mathrm{C}^{\alpha}$ atoms of the proximal and distal His residues in the $\alpha$ and $\beta$ heme sites (Fig. $3 a$ ) are identical at $14.3 \AA$ (not shown). We now discuss these features separately.

The $F_{\mathrm{o}}-F_{\mathrm{c}}$ OMIT electron-density maps and the hydrogenbonding networks in the distal pockets of this $2.0 \AA$ resolution aquometHb structure are shown in Fig. 3(a). For the $F_{\mathrm{o}}-F_{\mathrm{c}}$ OMIT electron-density maps, water molecules in the distal pockets were removed from the final model followed by restrained refinement using REFMAC prior to calculation of the maps. The hydrogenbonding networks are compared with those in the $1.6 \AA$ resolution structure of the related R-state horse aquometHb (Fig. 3b). Unlike those observed in the $\alpha$ heme sites of the other R-state human $\mathrm{Hb}$ structures ( $\mathrm{HbCO}$ and $\mathrm{HbO}_{2}$ in Fig. 1), the heme propionate of pyrrole ring 1 (next to Lys61) in the $\alpha$ subunit of R-state aquometHb points 'upwards' towards the distal His58. This apparently results in a movement of $4.7 \AA$ (when compared with the nitrite-derivative structure at $1.8 \AA$ resolution; PDB entry $3 \mathrm{~d} 7$ o; Yi et al., 2008) of the terminal amino $\mathrm{N}$ atom of Lys61 away from the heme to minimize a steric conflict while still maintaining a long-range electrostatic interaction ( $\sim .8 \AA$; not shown; Petsko \& Ringe, 2004) with a carboxylate $\mathrm{O}$ atom.

The distal His58 residue in the $\alpha$ subunit is hydrogen bonded to the Fe-bound $\mathrm{H}_{2} \mathrm{O}$ molecule and also forms a hydrogen-bonding interaction with a second $\mathrm{H}_{2} \mathrm{O}$ molecule that in turn hydrogen bonds to both propionate groups; this presumably provides stabilization to this 'locked' conformation. In addition, the His 45 residue (CD loop area) also hydrogen bonds to one of the propionates in this conformation. The bulk features of this hydrogen-bonding network in the $\alpha$ subunit are similar to those observed in horse aquometHb (Robinson et al., 2003) shown in Fig. 3(b), with the exception that the Lys61 in the

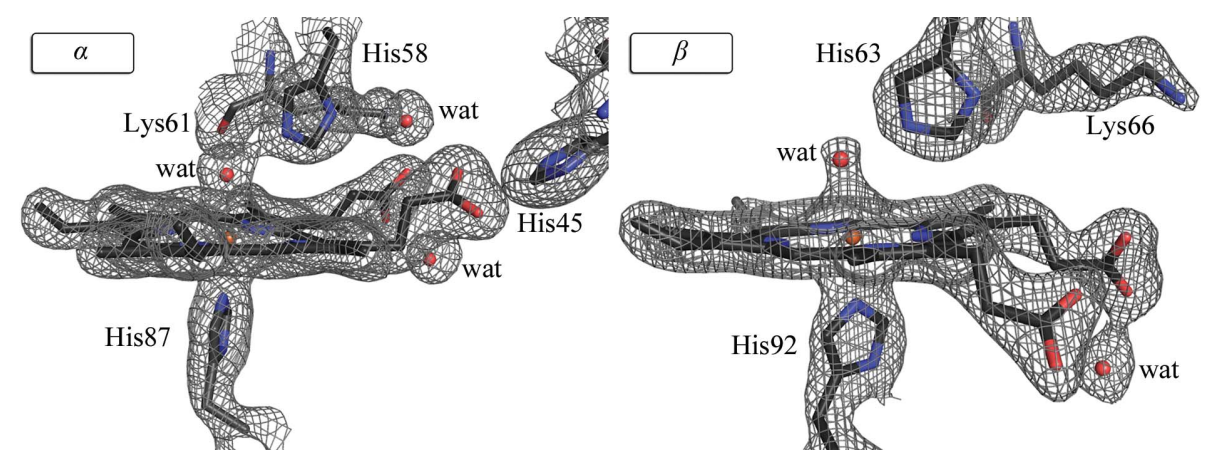

Figure 2

$2 F_{\mathrm{o}}-F_{\mathrm{c}}$ electron-density maps (absolute level $0.26 \mathrm{e} \AA^{-3}$ ) and final models of the heme active sites of the R-state aquometHb structure. 
R-state human aquometHb structure is not directly hydrogen bonded to the fixed water molecule that is hydrogen bonded to the heme propionates. Interestingly, the propionate groups in the T-state human aquometHb structure adopt a 'one up and one down' conformation (Liddington et al., 1992). In addition, fewer hydrogen bonds as well as a weaker hydrogen-bonding network are apparently present in the $\alpha$ heme site of the T-state metHb crystal structure (Fig. 3c).

The distal His63 residue in the $\beta$ subunit of human aquometHb is hydrogen bonded to the Fe-bound $\mathrm{H}_{2} \mathrm{O}$ ligand. However, and unlike in the $\alpha$ subunit, no additional hydrogen bonding to a fixed solvent molecule was observed. The Lys66 residue forms a salt bridge with one of the propionates, which in turn forms a strong hydrogen bond to a fixed $\mathrm{H}_{2} \mathrm{O}$ molecule at the proximal side of the heme. In contrast, one of the propionates in R-state horse aquometHb is in the 'up' position and the distal His63 residue also forms a second hydrogen bond to a solvent $\mathrm{H}_{2} \mathrm{O}$ molecule (Fig. $3 b$ ). The two additional water molecules in the $\beta$ heme site of the T-state human aquometHb structure, when compared with the two R-state aquometHb structures in Fig. 3, provide a stronger hydrogen-bonding network in this T-state $\beta$ site.

We speculate that the 'locked' conformation in the $\alpha$ subunit restricts movement of $\mathrm{H}_{2} \mathrm{O}$ in and out of the distal pocket. In contrast, the 'open' conformation in the $\beta$ subunit is likely to ease this restriction, thus increasing the motion of the $\mathrm{H}_{2} \mathrm{O}$ molecule at this $\beta$ site. However, we are hesitant to extrapolate this crystallographic result and speculation to the solution behavior of human aquometHb. In the reported R-state horse aquometHb and T-state human aquometHb structures (Fig. 3) the propionate groups in ring 4 of the $\beta$ hemes point 'upwards' towards the distal His63 residues and

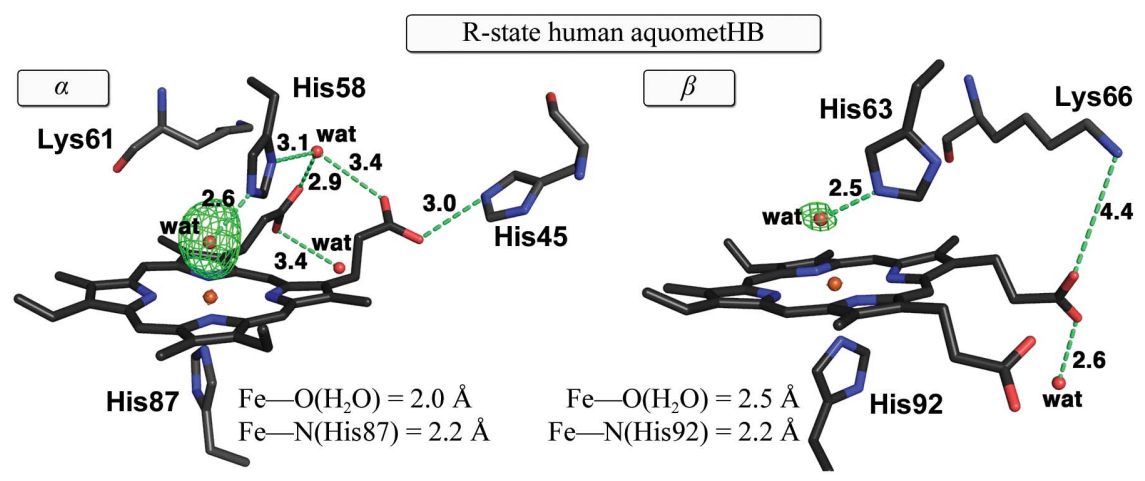

(a)

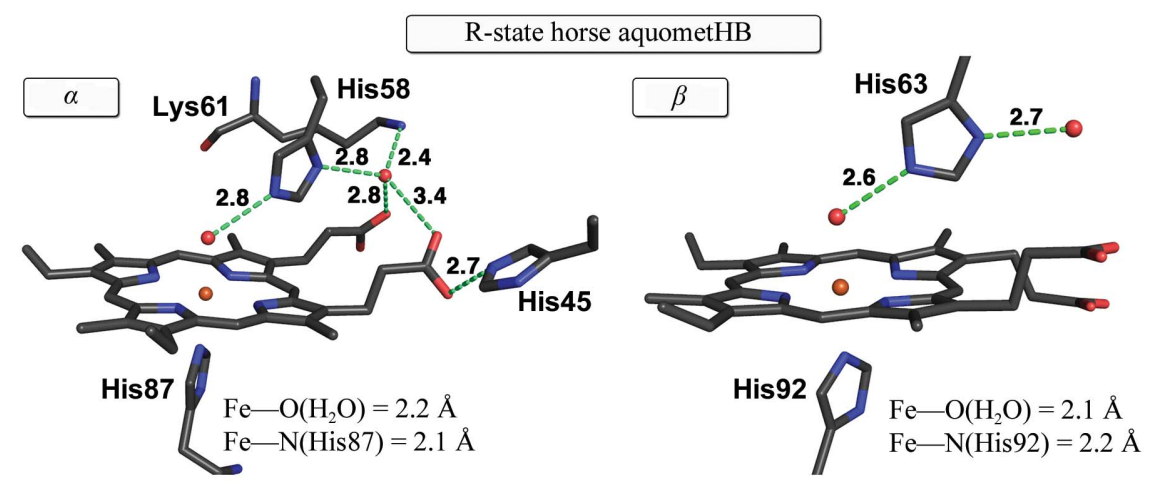

(b)

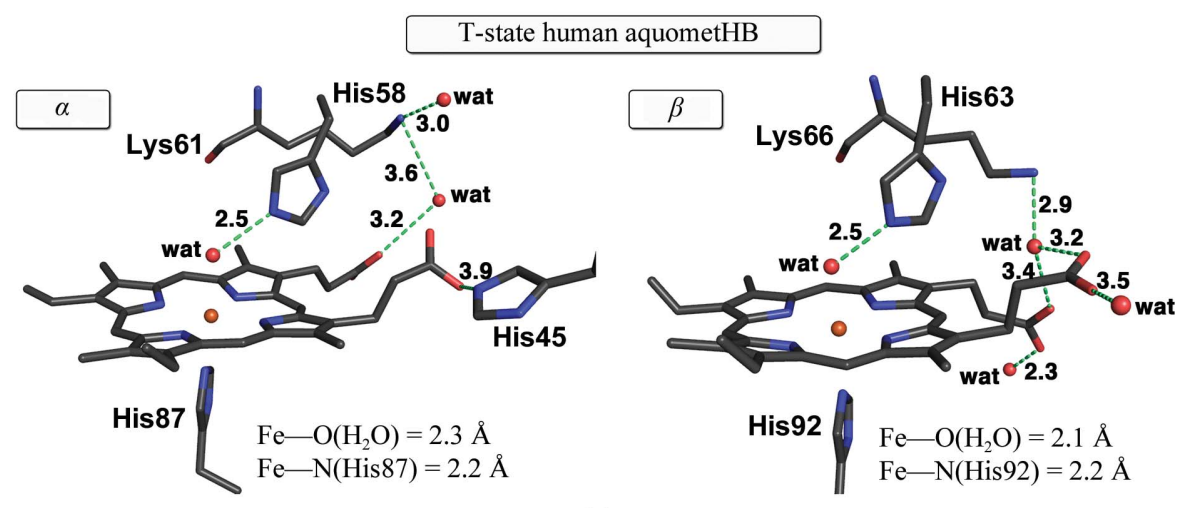

(c)

Figure 3

(a) $F_{\mathrm{o}}-F_{\mathrm{c}}$ OMIT electron-density map (absolute level $0.27 \mathrm{e} \AA^{-3}$ ) and final models of the heme active sites of R-state human aquometHb (PDB entry $3 \mathrm{p} 5 \mathrm{q}, 2.0 \AA$ resolution). (b) Models of R-state horse aquometHb (PDB entry 1ns9, $1.6 \AA$ resolution; Robinson et al., 2003). (c) Models of T-state human aquometHb (PDB entry 1hgb, 2.1 Å resolution; Liddington et al., 1992). 
we speculate that this may provide some stabilization of the $\mathrm{H}_{2} \mathrm{O}$ ligands.

The $\mathrm{Fe}-\mathrm{O}\left(\mathrm{H}_{2} \mathrm{O}\right)$ distance of $2.2 \AA$ in the $\alpha$ subunit of R-state human aquometHb is similar to that determined in other aquometHb structures [e.g. $2.2 \AA$ in R-state horse aquometHb (PDB entry 1ns9; Robinson et al., 2003) and $2.3 \AA$ in T-state human aquometHb (PDB entry 1hgb; Liddington et al., 1992)]. The related $\mathrm{Fe}-\mathrm{O}\left(\mathrm{H}_{2} \mathrm{O}\right)$ distance of $2.5 \AA$ in the $\beta$ subunit is longer than that in the $\alpha$ subunit and may reflect a weaker $\mathrm{Fe}-\mathrm{O}\left(\mathrm{H}_{2} \mathrm{O}\right)$ stabilization in the distal pocket and a resulting reduced population of fixed $\mathrm{H}_{2} \mathrm{O}$ at this site.

We are grateful to the Oklahoma Center for the Advancement of Science and Technology (HR09-081 to GBR-A) for funds to carry out this research. We are also grateful to Dr Martin Safo (Virginia Commonwealth University, USA) for introducing us to human $\mathrm{Hb}$ isolation and crystallization techniques.

\section{References}

Adams, P. D., Grosse-Kunstleve, R. W., Hung, L.-W., Ioerger, T. R., McCoy, A. J., Moriarty, N. W., Read, R. J., Sacchettini, J. C., Sauter, N. K. \& Terwilliger, T. C. (2002). Acta Cryst. D58, 1948-1954.

Biswal, B. K. \& Vijayan, M. (2002). Acta Cryst. D58, 1155-1161.

Chan, N. L., Kavanaugh, J. S., Rogers, P. H. \& Arnone, A. (2004). Biochemistry, 43, 118-132.

Chen, V. B., Arendall, W. B., Headd, J. J., Keedy, D. A., Immormino, R. M., Kapral, G. J., Murray, L. W., Richardson, J. S. \& Richardson, D. C. (2010). Acta Cryst. D66, 12-21.
DeLano, W. L. (2002). PyMOL. http://www.pymol.org.

Engh, R. A. \& Huber, R. (1991). Acta Cryst. A47, 392-400.

Kiese, M. \& Plattig, K. H. (1959). Naunyn Schmiedebergs Arch. Exp. Pathol. Pharmakol. 235, 373-380.

Ladner, R. C., Heidner, E. J. \& Perutz, M. F. (1977). J. Mol. Biol. 114, 385-414.

Liddington, R., Derewenda, Z., Dodson, E., Hubbard, R. \& Dodson, G. (1992). J. Mol. Biol. 228, 551-579.

Mauk, M. R. \& Mauk, A. G. (1982). Biochemistry, 21, 4730-4734.

Monod, J., Wyman, J. \& Changeux, J. P. (1965). J. Mol. Biol. 12, 88-118.

Murshudov, G. N., Skubák, P., Lebedev, A. A., Pannu, N. S., Steiner, R. A., Nicholls, R. A., Winn, M. D., Long, F. \& Vagin, A. A. (2011). Acta Cryst. D67, 355-367.

Park, S.-Y., Yokoyama, T., Shibayama, N., Shiro, Y. \& Tame, J. R. (2006). J. Mol. Biol. 360, 690-701.

Perutz, M. F., Rossmann, M. G., Cullis, A. F., Muirhead, H., Will, G. \& North, A. C. (1960). Nature (London), 185, 416-422.

Petsko, G. A. \& Ringe, D. (2004). Protein Structure and Function, p. 11. London: New Science Press.

Pflugrath, J. W. (1999). Acta Cryst. D55, 1718-1725.

Robinson, V. L., Smith, B. B. \& Arnone, A. (2003). Biochemistry, 42, 10113 10125.

Rockwood, G. A., Armstrong, K. R. \& Baskin, S. I. (2003). Exp. Biol. Med. 228, 79-83.

Safo, M. K. \& Abraham, D. J. (2003). Methods Mol. Med. 82, 1-19.

Safo, M. K. \& Abraham, D. J. (2005). Biochemistry, 44, 8347-8359.

Safo, M. K., Burnett, J. C., Musayev, F. N., Nokuri, S. \& Abraham, D. J. (2002). Acta Cryst. D58, 2031-2037.

Titov, V. Y. \& Petrenko, Y. M. (2005). Biochemistry, 70, 473-483.

Vagin, A. \& Teplyakov, A. (2010). Acta Cryst. D66, 22-25.

Winn, M. D. et al. (2011). Acta Cryst. D67, 235-242.

Yi, J., Safo, M. K. \& Richter-Addo, G. B. (2008). Biochemistry, 47, 8247-8249.

Yonetani, T. \& Laberge, M. (2008). Biochim. Biophys. Acta, 1784, 1146-1158. 